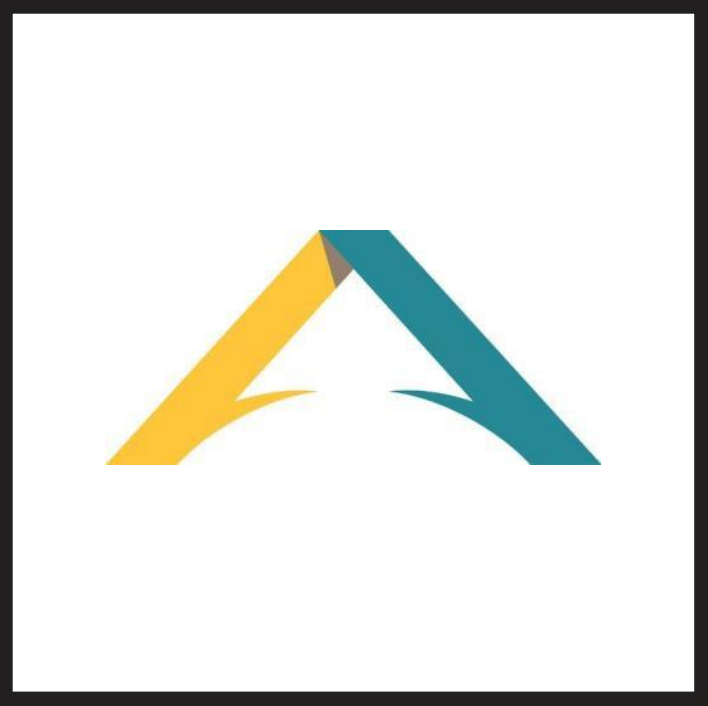

Revista

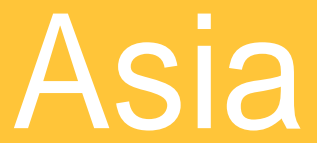

América

Latina

ISSN 2524-9347

Grupo de Estudios sobre Asia y América Latina Instituto de Estudios de América Latina y el Caribe Universidad de Buenos Aires

(c)

EU

DE BA 


\section{ASEAN: ¿REGLA O EXCEPCIÓN?}

ASEAN: ¿NORM OR RARITY?

\section{Catalina Mas}

Universidad de Buenos Aires

catalina.mas22@gmail.com

RESUMEN: El presente trabajo tiene como objetivo explorar la aplicabilidad de dos de las teorías de la integración regional más relevantes (neofuncionalismo e intergubernamentalismo) a la Asociación de Naciones del Sudeste Asiático (ASEAN, por sus siglas en inglés). En este sentido, se buscará encontrar, a través de fuentes primarias y secundarias, las características más relevantes de ambas corrientes. Se les prestará especial atención a los acuerdos plasmados en forma de instrumentos legales, ya que se constituyen como la materialización de estos. De este modo, es posible medir la frecuencia, eje temático y nivel de especialización de cada acuerdo para testear si se trata de instrumentos de naturaleza intergubernamental o, por el contrario, se puede observar algún nivel de spillover con el correr de los años.

Palabras clave: ASEAN, integración regional, cooperación, neofuncionalismo, intergubernamentalismo.

ABSTRACT: The following article seeks to explore the applicability of two of the most relevant regional integration theories (neofunctionalism and intergovernmentalism) regarding the Association of Southeast Asian Nations (ASEAN). To do so, it will try and find the key elements of each theory present in its institutional functioning through primary and secondary sources. Special attention will be paid to the agreements between the parties in the form of legal instruments since they are the materialized form. In this manner, it is possible to monitor and measure the frequency, topic, and level of specialization of each agreement, to test whether they have an intergovernmental nature, or, on the contrary, there is a spillover effect over the years.

Key Words: ASEAN, regional integration, cooperation, neofunctionalism, intergovernmentalism. 


\section{Introducción}

La Asociación de Naciones del Sudeste Asiático (ASEAN, por sus siglas en inglés) ha pasado por diversos procesos de profundización en materia de cooperación. En este sentido, la agenda fue mutando desde los objetivos securitarios para hacerle frente a Vietnam y posteriormente China, hasta la profundización en la cooperación económica y social. El pasaje desde 1967, con la firma del primer tratado que instauró un trato preferencial entre sus miembros, hasta la cumbre de Singapur en 1993, que definió las bases para una zona de libre comercio, demostrarían la importancia creciente que supuso la integración regional para sus miembros (Laursen, 2010). Actualmente, la organización posee principios plasmados en tratados, una organización tripartita y un organigrama que parecería apuntar hacia una mayor cooperación en áreas más abarcativas de la política regional (Laursen, 2010; Min-hyung, 2011).

En la actualidad, la ASEAN está posicionada como un actor estratégico en la arena internacional, abriendo la posibilidad de dialogar con otros actores de forma conjunta e inaugurando foros ampliados como ASEAN+3. Dependiendo de la perspectiva de análisis con la que se decida analizar a ASEAN se lo podría considerar -desde la perspectiva securitaria- como un elemento de balancing regional mostrando un contrapeso con China, o bien -desde el punto de vista económico- como un bloque que permite posicionar mejor a la región en términos económicos y comerciales (Börzel, 2015; Min-hyung, 2011).

Sin embargo, el hecho de mostrar una mayor cohesión no eclipsa lo que los académicos han denominado el "ASEAN way", una forma de diálogo y prevención de conflictos entre los integrantes que salvaguarda la autonomía nacional de cada uno de los Estados parte. Tanto su relevancia en la región y a nivel sistémico como su particularidad generan la necesidad de entender más y mejor el fenómeno del "ASEAN way". En este sentido, es interesante revisitar las teorías de la integración regional a la luz de este caso para así testear su capacidad explicativa.

\section{Metodología}

$\mathrm{Al}$ momento de analizar las experiencias de integración regional, no es menor el hecho de que las teorías clásicas hayan surgido a la luz de la experiencia europea. Se trata de teorías que pretenden ser de rango entre medio y alto, siendo altamente generalizables (Malamud, 2011). Asimismo, han surgido varias críticas y teorías alternativas como la de gobernanza (Börzel, 2015). Si bien estas no son incompatibles con ciertas corrientes de las teorías clásicas, generan aportes significativos que nos pueden ayudar a comprender mejor las dinámicas de interacción entre los actores. 


\section{ASEAN: ¿regla o excepción? \\ CATALINA MAS}

El fin del presente trabajo será explorar el nivel de aplicabilidad de las principales teorías clásicas de la integración (neofuncionalismo e intergubernamentalismo) para tratar de responder cuál se adecúa más al caso de ASEAN. A tal fin se revisarán, en primer lugar, los principios generales de los dos grandes cuerpos de teorías de la integración regional y los aportes de la perspectiva de gobernanza (Börzel, 2015). En segundo lugar, se intentará aplicar los elementos esenciales de cada cuerpo teórico al caso de ASEAN siguiendo las particularidades organizativas, los acuerdos logrados y las experiencias históricas.

En este sentido, se utilizará una metodología mixta cuali y cuantitativa. Por un lado, se revisarán fuentes primarias publicadas por ASEAN en su página oficial, por ejemplo, el organigrama y los tratados y principios explicitados en su tratado de conformación. A su vez, se consultarán fuentes secundarias con respecto a los "valores asiáticos" -orden y disciplina- de la región (Del Río Martínez, 2018), la historia y el funcionamiento de ASEAN. Finalmente, se realizará un análisis cuantitativo del desarrollo de instrumentos legales y acuerdos teniendo como eje la temporalidad a fin de distinguir si hubo mutación en los temas más salientes y testear la posibilidad de encontrar un fenómeno de spillover (Schimmelfennig y Rittberger, 2006).

El presente trabajo es una primera aproximación que deberá ser complementada con otros monitoreos a futuro. Se tomarán como fuente el listado de acuerdos e instrumentos legales ASEAN ya clasificados en las tres comunidades (económica, político-securitaria y sociocultural). Se le asignará un eje temático y, posteriormente, se operacionalizarán en cuanto a su grado de especificidad en dos tipos: 1. referidos a los marcos organizacionales de la ASEAN; 2. referidos a la resolución sustancial de una temática específica.

\section{Aproximaciones teóricas clásicas y aportes de Gobernanza}

\section{III.1. Neofuncionalismo}

El neofuncionalismo se considerará a la luz de las contribuciones de varios autores supranacionalistas (especialmente institucionalistas sociales y legales), ya que es posible pensar en estas teorías como las sucesoras directas de la teoría neofuncionalista clásica fundada por Haas (Stroby Jensen, 2016). El neofuncionalismo asume que el mismo mecanismo de la integración produce una mayor integración a través de, al menos, cuatro mecanismos: spillover funcional, spillover político, spillover institucional, y la sociabilización de elites. Considera como premisa básica que "la cooperación en un área de la política crea presión en otras áreas, posicionándolas en la agenda política" (Schimmelfennig y Ritterberg, 2005, p. 84). Al contrario de los realistas e intergubernamentalistas, consideran que el proceso de integración no debería ser considerado como un juego de suma cero bajo el cual los Estados pierden frente 
al fortalecimiento de otros. Adicionalmente, interpreta que este proceso es semiautomático (o path-dependent), donde los grupos de interés, actores no gubernamentales y lobbies juegan un rol sumamente importante.

La noción de "semiautomático" o path-dependent es mejor entendida a través del concepto de spillover. Como se ha mencionado anteriormente, "refiere al proceso mediante el cual los miembros de un proyecto de integración (...) intentan resolver cuestiones insatisfechas recurriendo a la colaboración en otro sector relacionado" (Schmitter, 1969, p. 162). Estos procesos de spillover pueden ser clasificados en tres tipos. El primero es el spillover funcional, que refiere al mecanismo mediante el cual cada paso en la cooperación lleva a otro. Un ejemplo de esto es la cooperación en el área económica (Stroby Jensen, 2016). El segundo es el spillover político, en este caso son las elites nacionales y lobbies quienes impulsan la cooperación con la excusa de que es necesaria para resolver problemas específicos.

El tercer tipo es el spillover institucional, según el cual existen instituciones supranacionales que habilitan -o no- el espacio para el spillover político a partir de externalidades positivas o negativas generando incentivos para extender y profundizar la integración dando cuenta de sus intereses comunes (Schimmelfennig y Rittberger, 2006). Sumado a los tres mecanismos de spillover hay un cuarto elemento que facilita la cooperación: la socialización de la elite. Esta está sumamente relacionada con el spillover político y refiere a los cambios en las alianzas y lealtades de actores claves del plano nacional al supranacional. Esto llevaría la conformación de grupos leales a las instituciones supranacionales (Stroby Jensen. 2016).

\section{III.2. Intergubernamentalismo}

El intergubernamentalismo nació como teoría a mediados de la década de 1960 como respuesta a la teoría neofuncionalista. Algunos de los aportes dentro de esta línea son el enfoque de política doméstica, el locking-in de los Estados y el intergubernamentalismo liberal (Cini, 2016). Los académicos que suscriben esta teoría están mayormente relacionados con la escuela neorrealista de relaciones internacionales, con presupuestos e hipótesis similares exceptuando el intergubernamentalismo liberal. En primer lugar, consideran que la política internacional, incluyendo los proyectos de integración regional, son un juego de suma cero con ganadores y perdedores. En segundo lugar, creen en la lógica de actuación de los Estados bajo los principios de autoayuda en la que cada Estado buscará maximizar su posición en una arena internacional anárquica (Cini, 2016; Morgenthau, 1985; Pollack, 2012).

Sin embargo, los teóricos neorrealistas, en contraste con los realistas clásicos, creen que la cooperación entre Estados puede surgir en momentos en los que es conveniente para los intereses de los Estado miembro, ayudando a 


\section{ASEAN: ¿regla o excepción? \\ CATALINA MAS}

reducir los niveles de anarquía y los costos de cooperación a largo plazo. "La cooperación es esencialmente conservadora y pragmática. Descansa en las premisas de que para resolver problemas en común son necesarias soluciones en común" (Cini, 2016, p. 67). El intergubernamentalismo cree que cada Estado negocia y sopesa pros y contras en cada paso hacia la profundización del proceso de integración. Como menciona Cini (2016), los Estados no transfieren soberanía como lo consideran los neofuncionalistas, sino que la aúnan o crean un pool de soberanía en distintas áreas de políticas públicas.

Si bien el intergubernamentalismo sigue siendo una teoría relevante, ha sido criticado por varios académicos. El mayor de los cuestionamientos refiere a que la mayoría de las corrientes desestiman el plano doméstico que puede tener grandes consecuencias en términos de agenda-setting y decisión de las elites de cada Nación. Es decir, que este componente es otro de los cálculos que debería tener en cuenta la teoría. El enfoque de política doméstica y el intergubernamentalismo liberal miran más en profundidad este asunto. Analizan indicadores sobresalientes en el contexto doméstico al momento en que se producen las grandes oleadas de integración (expansión y profundización), intentando explicar las decisiones tomadas de acuerdo con el plano internacional y los incentivos nacionales.

Adicionalmente, el enfoque de locking-in de los Estados ayuda a analizar el efecto bola de nieve de cooperación presente en las teorías neofuncionalistas. Consideran que los factores institucionales son importantes y que, pasado un punto, los Estados se ven "encerrados" dentro de una estructura de cooperación institucional por fuera de la cual no pueden o no tienen incentivo para actuar. Este enfoque, a diferencia de los neofuncionalistas, considera que los Estados siguen siendo el principal actor que decide continuar con el proceso de pooling de soberanía basado en la racionalidad instrumental de un gobierno ya que termina generando externalidades positivas. En este enfoque la cooperación sigue siendo racional y pragmática. Finalmente, el intergubernamentalismo cree, a diferencia del neofuncionalismo, que es una teoría ontológicamente reproductiva, cree que los grandes eventos son los responsables en el cambio en las instituciones. No hay un costado transformativo y evolutivo en las instituciones, no son pathdependent, los Estados tienen que promover los cambios institucionales basados en cambios en el equilibrio y los incentivos pragmáticos.

\section{III.3. Gobernanza}

El enfoque de gobernanza les da igual importancia a los actores estatales como no estatales. Esto implica tener en cuenta no solo las redes institucionales formales, sino también las informales (Börzel, 2015). Este enfoque prioriza tanto a los actores como al proceso y progreso en la relación de los actores. En este sentido, este nuevo aporte sigue las directrices de los principios comprendidos 
en el neofuncionalismo. El enfoque de gobernanza generalmente distingue tres tipos de reglas institucionalizadas: jerárquicas, competitivas (mercados) y de negociación (redes de contactos) (Börzel, 2015). Las últimas dos son no jerárquicas y son de aplicación voluntaria; esto implica que los conflictos de interés son resueltos mediante negociaciones y no a través del enforcement de leyes previamente establecidas.

Este aporte se centra en el impacto que tiene el estilo de gobernanza sobre las instituciones y sus formas de comportarse, coordinar, expandir y/o profundizar los procesos de integración regional y cooperación. En este sentido, el enfoque comparte puntos en común con las teorías más racionalistas, como el cálculo racional de las teorías neorrealistas e intergubernamentalistas sobre los incentivos para cooperar. Asimismo, comparte el enfoque bipartido entre los actores estatales y no estatales como las teorías neofuncionalistas. Sin embargo, no solo tiene puntos en común con los enfoques racionalistas, sino que también es compatible con las perspectivas constructivistas al tener en cuenta las percepciones e interpretaciones de los actores involucrados.

De esta manera, este enfoque se nutre de las críticas y avances que cada cuerpo de teorías de la integración regional fue experimentando en el recorrido histórico de la construcción y testeo de conceptos. Llega a ser una teoría, en cierto sentido, integradora. Reconcilia distintos conceptos de los dos cuerpos de teorías racionalistas predominantes en la literatura de la integración regional, sumándole elementos del constructivismo que ha sido relegado por parte de la mayoría de los académicos de este fenómeno.

\section{Particularidad asiática}

Como se mencionó anteriormente, la ASEAN es un proyecto de integración regional que surgió a la luz de dilemas securitarios en la zona con el fin de servir de balance frente a la preponderancia de Indonesia, y posteriormente de mantenerse neutros frente a las vicisitudes de la Guerra Fría siendo el Sudeste Asiático una zona álgida en la disputa entre los dos hegemones (Estados Unidos y la Unión Soviética) (Börzel, 2015). Actualmente, a su vez, el hecho de que todos los países del Sudeste Asiático ${ }^{1}$ se hayan unido les permite negociar y posicionarse de mejor manera frente a China y los mercados internacionales. En este sentido, más allá de la declaración de neutralidad, se utilizó el fortalecimiento de la industria y la economía para lograr ser un pivot de negociación y fortalecer sus posiciones (Laursen, 2010).

En términos de funcionamiento, la misma ASEAN se autodenomina intergubernamentalista. Esto se refleja en un liderazgo débil sin preponderancia

\footnotetext{
1 Timor Oriental está actualmente negociando su incorporación a la ASEAN.
} 


\section{ASEAN: ¿regla o excepción? \\ CATALINA MAS}

de un líder político (Laursen, 2010), mecanismos de resolución de disputas a través de la negociación, respetando la soberanía de cada una de las partes. Esto se observa en la declaración de principios comprendida en el Tratado de Amistad y Cooperación de 1976:

Respeto mutuo por la independencia, soberanía, igualdad, integridad territorial e identidad nacional de todos los miembros; El derecho de cada estado a guiar su existencia nacional libre de cualquier coerción, interferencia o subversión exterior; No-interferencia en los asuntos internos de otro país.; Solución de las diferencias o disputas por medios pacíficos; Renuncia a la amenaza o uso de la fuerza; Cooperación efectiva entre los países miembros. (Association of Southeast Asian Nations, 1976)

Todo esto en su conjunto conforma lo que la mayoría de los académicos han bautizado como la $A S E A N$ way. A simple vista, esta organización debería ser analizada bajo el lente del intergubernamentalismo, ya que se trata de un foro institucionalizado de negociación donde son los mismos mandatarios estatales los que se ponen de acuerdo en seguir líneas similares de políticas en distintas áreas a su vez que aplican una tarifa interna entre ellos. Sin embargo, a su vez, a lo largo de los años se han abierto canales de comunicación que le permiten un rol mayor a actores y grupos de interés no estatales como lo son las cámaras de comercio (ASEAN Chambers of Commerce and Industry), las Iniciativas Interministeriales de cooperación (ASEAN University Network) la asociación portuaria o los centros de estudios estratégicos (ASEAN Center for Energy) y otras organizaciones no gubernamentales que han establecido relación (ASEAN, 2015).

Asimismo, el hecho de que resolvieran las disputas a través de la negociación podría ser un símbolo de los denominados "valores asiáticos" (Lee, 1998) y no un diagnóstico con respecto a la mecánica de interacción de ASEAN mismo, esto escaparía al objeto de estudio de una teoría racionalista como lo es el intergubernamentalismo. Esto indicaría la importancia de intentar analizar el fenómeno bajo el lente del neofuncionalismo para entender la interacción con actores no estatales y conjugarlo con teorías con un enfoque más constructivista para comprender si se debe a la percepción y construcción identitaria de los actores. Los "valores asiáticos" integrarían el desarrollo económico y la disciplina social, esto indica que los asiáticos poseen un espíritu de equipo que les permite cooperar. Esto se ve plasmado en los conceptos de musjawarah (consulta) y mufakat (consenso), que definen al respeto por la soberanía y la no interferencia en asuntos internos (Min-hyung, 2011).

La responsabilidad social y el deber de los mandatarios con sus pueblos de acuerdo con el contrato social asiático permitiría confiar en la negociación y a su vez podría poner una traba a la posibilidad de delegar soberanía en un órgano supranacional, ya que violaría este contrato. Sin embargo, Lee (1998) 
desmitifica este concepto de los "valores asiáticos" definiéndolo como un artificio ex post a fin de justificar políticas represivas y como modo de contraposición ética frente a occidente.

En términos neofuncionalistas, la característica que más llama la atención es la convivencia de tres comunidades distintas: la comunidad económica, político-securitaria y sociocultural. Esto podría ser un síntoma del efecto spillover que introducen los neofuncionalistas, ya que para poder seguir cooperando en un área económica es necesario profundizar la cooperación en otra área como la política. Tal y como lo indica Laursen (2010), la Declaración de Bali de 2003 establece los tres pilares o comunidades como mutuamente interdependientes y reforzantes. Esto implicaría una distribución creciente en la cantidad de acuerdos de un área con respecto a otras. Al analizar la distribución temporal de los acuerdos, sin embargo, este fenómeno no se verifica. La dimensión económica tiene una preponderancia del $84 \%$ con respecto al resto de las comunidades (12\% comunidad política y $3 \%$ la comunidad sociocultural). No se encuentra un crecimiento orgánico debido al efecto del spillover, sino que los acuerdos políticos se generan en oleadas al igual que los culturales y económicos.

Con respecto a los económicos, hay tres picos en los acuerdos en 1998, 2005 y 2010. Tanto en 1998 como 2010 coinciden con los dos años inmediatamente posteriores a estallidos de crisis económicas regionales (1997) y globales (2008). Esto coincidiría con la hipótesis intergubernamental de oleadas de voluntad política por cooperar a fin de mejorar la posición a nivel sistémico de los países. Es cierto que la frecuencia de cumbres realizadas se ha incrementado (Min-hyung, 2011), por lo que se verifica un aumento en la interacción de las partes, lo cual podría indicar una creciente socialización de las elites. Esto podría deberse, sin embargo, a los efectos de la globalización que genera incentivos racionales a cooperar, y no necesariamente a un efecto neofuncionalista de socialización. A su vez, ASEAN es una organización internacional que ya lleva más de 52 años en la arena política y que cuenta con precedentes de interacción en foros de cooperación como el Foro de Cooperación Económica Asia-Pacífico (APEC por sus siglas en inglés), donde las elites -no solo estatales-interactúan. A pesar de esto, no se habilitó una gran profundización en la integración regional, por lo que este mecanismo que llevaría a la profundización de esta no sería explicativo en el caso de ASEAN.

En lo que respecta al tratamiento específico de cada una de las comunidades, se puede observar que la preponderancia de la comunidad económica no es solamente cuantitativa, sino también cualitativa. Mientras que en la comunidad política la mayoría de los acuerdos son a fines de crear la estructura organizativa de ASEAN, en la comunidad económica se llegan a consensos sustanciales con respecto a temas específicos y muy sensibles. En la cuestión sociocultural, también hay una prevalencia del $62 \%$ en lo que respecta a consensos sobre temas específicos, sin embargo, tan solo se han logrado firmar 


\section{ASEAN: ¿regla o excepción? \\ CATALINA MAS}

8 instrumentos dentro de esta comunidad ( $3 \%$ del total). A su vez, se puede observar que la comunidad económica tiene una gran fragmentación en cuanto a ejes temáticos sobre los que se han acordado cuestiones, siendo los más salientes el comercio, seguido por la provisión de servicios y transporte. La comunidad política, por su parte, tiene una preponderancia en cuestiones referentes a las formalidades, principios y fondos de cooperación del ASEAN mismo. Paralelamente, la comunidad sociocultural tiene como prioridad cuestiones referentes a la educación, la ecología y la cooperación humanitaria. De esta forma, se podría concluir que las teorías neofuncionalistas no son las más indicadas para comprender la totalidad del proceso de integración de ASEAN. Sin embargo, nos aportan otro enfoque que no habría que perder de vista frente a la preminencia de las teorías intergubernamentalistas.

Finalmente, podemos considerar la teoría de la gobernanza como la síntesis que mejor se puede llegar a explicar el caso de ASEAN. La misma logra incluir tanto la perspectiva racionalista como la constructivista. A su vez, entiende la mecánica orgánica de más y mayor integración como derivado de las instituciones y actores (estatales y no estatales) propios de las teorías neofuncionalistas. Esto mismo se verifica en el entendimiento de la prevalencia de las formas de toma de decisiones entre actores estatales y las características predominantemente intergubernamentalistas. Comprende, por un lado, que para poder cooperar no es necesario solo una balanza de beneficios mayor a los peligros, sino también las expectativas de los actores con respecto al resto de los estados parte. Esto se verifica con las oleadas de ingresos a países que coincide con la pacificación o finalización de conflictos domésticos de parte de estos, lo cual permite una expectativa mayormente enfocada en la negociación y la confianza. A su vez comprende que, si bien los actores formalmente de veto son los Estados, también hay foros paralelos donde se conforman grupos de interés no estatales.

Asimismo, agrega la noción de los tres tipos de reglas institucionalizadas. ASEAN no posee un mecanismo de enforcement donde se prevea un tribunal o penalidades fijas por el no cumplimiento de los pactos o disputas, sino que elige la vía de la negociación entre partes respetando la soberanía de cada uno de los Estados por igual. De esta forma, se trataría de un tipo de regla no jerárquico con arreglo a negociar en el caso de disputas.

A su vez, la mayoría de los temas en agenda corresponden a la economía. De esta forma también habría cierta injerencia del tipo de regla de competencia u orientada al mercado. En este sentido, es posible pensar el aumento en la cooperación como consecuencia de la globalización, diversificando el tipo de actores internacionales, habilitando nuevos canales de comunicación y generando incentivos positivos para la cooperación regional frente a una mayor competencia internacional. Esto sigue las directrices que la teoría de la gobernanza indica (Börzel, 2015). 


\section{Conclusión}

Derivado de lo anterior, podemos concluir que el caso de ASEAN podría llegar a considerarse como uno de los más complejos hasta el momento. Conjuga elementos que se encuentran dispersos en distintas teorías desarrolladas hasta el momento. Debido a que el compendio teórico en lo que refiere a teorías de la integración de rango medio está mayormente inspirado en la experiencia europea, no hay una única teoría que refleje la plétora de elementos únicos que están presentes en el sudeste asiático. Es necesario avanzar hacia teorías más abarcativas y holísticas. El enfoque de gobernanza es, entonces, un ejemplo de las posibilidades teóricas que se encuentran más allá de los horizontes que dividen a racionalistas, liberales y constructivistas, a la vez que dividen a aquellos que ven a las organizaciones supranacionales como neofuncionalistas $o$ intergubernamentalistas.

Siguiendo los paradigmas clásicos, deberíamos descartar las teorías neofuncionalistas debido a que no se verifica la mayoría de sus premisas en la práctica ni habría indicadores de una profundización organizacional que lleva a pensar que en un futuro este sería el camino. Pero a su vez, tampoco se puede tomar a la teoría intergubernamental en su totalidad, ya que hay elementos únicos al caso del Sudeste Asiático que no son comprendidos por sus desarrollos teóricos, mayormente las cuestiones de mayor integración entre actores no estatales y las expectativas de los actores involucrados desde la perspectiva constructivista.

ASEAN es un caso en el que se encuentran distintos niveles de cooperación, momentos coyunturales y pragmáticos de expansión y profundización de la integración. Es una organización que posee un plan de desarrollo semineofuncionalista frente a las disputas, sin terminar de funcionar de esta manera ni siquiera de manera nominal. Este artículo ha buscado introducir la aplicación de algunas de las teorías básicas en la literatura de integración regional. Se deberá ahondar a futuro con más y mejores herramientas metodológicas, teóricas e información acerca del desarrollo efectivo del proceso de integración a medida que avance el desarrollo de la organización y los nuevos aportes teóricos sobre integración regional.

\section{Referencias bibliográficas}

Association of Southeast Asian Nations (1976). Treaty of Amity and Cooperation in Southeast Asia (TACSE). https://asean.org/treatyamity-cooperation-southeast-asia-indonesia-24-february-1976/ 
BÖRZEL, T. (2015). Theorizing Regionalism: Cooperation, Integration, and Governance. En Börzel, T.A. y Risse, T. (Eds.), Oxford Handbook of Comparative Regionalism (41-63). Oxford University Press.

CINI, M. (2016). Intergovernmentalism. En Cini, M. y Pérez-Solórzano Borragán, N. (Eds.), European Union Politics (65-78). Oxford University Press.

Del Río MARTíneZ, A. (2007). "La tercera ola democrática en Asia: explicación política para un fenómeno político". Actas del XII Congreso Internacional de ALADAA. Benemérita Universidad de Puebla, México.

JENSEN, C. S. (2000). Neofunctionalist theories and the development of the European social and labour market policy. Journal of Common Market Studies, Vol, 38/1, pp. 21-92

LAURSEN, F. (2010). Comparative Regional Integration: Europe and beyond. Ashgate: Farnham, Reino Unido.

LEAL-ARCAS, R. (2007). Theories of Supranationalism in the EU. Journal of Law in Society, Vol. 8, No. 1, pp. 83-113.

LEE, E. J. (1998). ¿Valores asiáticos como ideal de civilización? Nueva sociedad No. 155 (Mayo-Junio): 111-125.

Malamud, A. (2011). Conceptos, teorías y debates sobre la integración regional. Norteamérica, 6(2), 219-249.

MiN-HYUNG KIM (2011). Theorizing ASEAN Integration. Asian Perspective, Vol. 35, No. 3: 407-435.

Morgenthau, H. (1985). Politics among nations: The struggle for power and peace. New York: Knopf.

POLlack, M. A. (2012). Theorizing the European Union: realists, intergovernmentallist and institutionalist approaches. En Jones, E., Menon, A. y Weatherill, S. (eds), European Integration Theory. Oxford: Oxford University Press.

SCHIMMELFENNING F. y RITTBERGER B. (2006). Theories of European integration: assumptions and hypotheses. En Richardson, J. (Ed.), European Union. Power and policy-making (73-95). London: Routledge.

SCHMITTER, P. (1969). Three neofunctional hypotheses about international integration. International Organization, 23/1: 161-6.

Stroby Jensen, C. (2016). Neo-functionalism. En Cini, M. y Pérez-Solórzano Borragán, N. (Eds.), European Union Politics (53-64). Oxford University Press.

SwEET, A. S. (2012). Neofunctionalism and Supranational Governance (unabridged version). Faculty Scholarship Series. Paper 4628.

WeILER. J. (1981). The Community System: the Dual Character of Supranationalism. Yearbook of European Law, 1/1. 267-306 

